\title{
ANÁLISE DO NÍVEL DE SERVIÇO VIA SIMULAÇÃO POR EVENTOS DISCRETOS: ESTUDO DE CASO EM UMA REDE DE DROGARIAS
}

\author{
Pedro Henrique Resende Marques \\ Universidade Federal de Minas Gerais \\ Av. Antônio Carlos, 6627, Belo Horizonte, MG, Brasil. CEP: 31270-901 \\ pedrohmarqs@gmail.com \\ Beatriz Amâncio Sofal \\ Universidade Federal de Minas Gerais \\ Av. Antônio Carlos, 6627, Belo Horizonte, MG, Brasil. CEP: 31270-901 \\ beatrizsofal@yahoo.com \\ Guilherme Ribeiro Souza \\ Universidade Federal de Minas Gerais \\ Av. Antônio Carlos, 6627, Belo Horizonte, MG, Brasil. CEP: 31270-901 \\ guilhermers.eng@gmail.com \\ João Flávio de Freitas Almeida \\ Universidade Federal de Minas Gerais \\ Av. Antônio Carlos, 6627, Belo Horizonte, MG, Brasil. CEP: 31270-901 \\ joao.flavio@dep.ufmg.br
}

\section{RESUMO}

A demanda por serviços farmacêuticos de qualidade vem aumentando com o envelhecimento da população Brasileira. Um desbalanceamento entre a oferta de serviços pode tanto levar clientes para em longas filas, como gerar ociosidade de atendentes. O presente estudo de caso visa dimensionar o número de atendentes em unidades de uma farmácia por uma abordagem quantitativa via simulação por eventos discretos. Busca-se avaliar a agilidade de atendimento contemplando regras de priorizações de atendimento por perfil de cliente. As análises de desempenho avaliam cenários extremos, como baixa demanda ou horário de pico contemplando alterações em taxas de chegada e no número de funcionários. Embora o tempo médio em fila tenha aumentado de aproximadamente zero para 8 minutos, o nível de serviço da drogaria permaneceu acima dos $80 \%$, considerado como satisfatório para clientes e pelos gestores.

Palavra-chave: Serviços; Saúde; Farmácia; Simulação por eventos discretos; Simpy. 


\begin{abstract}
The demand for good pharmaceutical services has been increasing with the aging of the Brazilian population. An imbalance between service delivery can either lead customers to long queues, or lead to attendant idleness. This case study aims to size the number of attendants in drugstore units by a quantitative approach via discrete event simulation. We seek to evaluate the agility of care by contemplating rules of prioritization of care by customer profile. Performance reviews evaluate extreme scenarios, such as low demand or peak hours contemplating changes in arrival rates and the number of employees. Although the average queue time increased from approximately zero for 8 minutes, the pharmacy service level remained above $80 \%$ considered satisfactory to customers and managers.
\end{abstract}

Keywords: Service; Heathcare; Drugstore; Discrete event simulation; Simpy.

\title{
Como Citar:
}

MARQUES, Pedro Henrique Resende; SOFAL, Beatriz Amâncio; SOUZA, Guilherme Ribeiro; ALMEIDA, João Flávio de Freitas. Análise do nível de serviço via simulação por eventos discretos: estudo de caso em uma rede de drogarias. In: SIMPÓSIO DE PESQUISA OPERACIONAL E LOGÍSTICA DA MARINHA, 19., 2019, Rio de Janeiro, RJ. Anais [...]. Rio de Janeiro: Centro de Análises de Sistemas Navais, 2019.

\section{INTRODUÇÃO}

As operações de serviços oferecidos por drogarias e farmácias possuem componentes cuja dinâmica pode ser descrita por sistemas de filas. O estudo de modelos quantitativos, como a teoria de filas, são base para análise de fatores intrínsecos aos sistemas e aos seus agentes [1]. No entanto, a simulação por eventos discretos tornase relevante à medida em que as operações se tornam mais dinâmicas e os tempos de operações são representadas por distribuições de probabilidade cujas propriedades teóricas são menos conhecidas [2]. No meio farmacêutico, o uso de ferramentas de simulação favorece o planejamento de capacidade e a melhoria da gestão [3].

Este artigo apresenta um estudo abordando a operação de unidades de uma rede de farmácias. Os resultados deste estudo podem ser generalizados e replicados a outras unidades apresentando grande contribuição tanto à literatura de estudos de caso como na prática, visto que o Conselho Federal de Farmácia estima a presença de mais de 82 mil farmácias e drogarias no Brasil [4]. Assim, propõe-se 4 cenários com diferentes taxas de chegada de clientes e modos de operação de maneira a observar o desempenho do atendimento. O nível de serviço ao cliente é um fator estratégico de diferenciação da empresa de demais redes farmacêuticas. Ademais, o estudo analisa cenários resultantes de alterações nos níveis de serviço da farmácia. Este deve manter-se adequado aos padrões de qualidade e alinhado ao modelo de negócio da organização.

Embora os níveis de serviço possam ser quantificados, o conceito que define a qualidade de atendimento é abstrato e resultante de fatores como urbanização, mudanças demográficas, socioeconômicas e tecnológicas, e ainda o aumento da so- 
fisticação dos consumidores [5]. Para avaliar o nível de serviço de atendimentos, recorre-se à simulação por eventos discretos. O método permite a modelagem e análise de problemas reais, com o qual é possível descrever e prever o comportamento de um sistema por meio da avaliação de cenários. A adoção da simulação tem sido crescente tanto no meio acadêmico como no meio industrial [6], enquanto isso, o setor farmacêutico também se desenvolve.

A pirâmide etária do Brasil tem apresentado um aumento do envelhecimento da população em relação a períodos passados, como demonstrado nas Figuras 1 e 2. Tal fato implica aumento da demanda por drogarias [7]. Estudos que avaliam o perfil do consumidor deste setor apontam que o consumidor realiza, em média, uma compra em farmácias a cada 15 dias [8]. Sobre a percepção da qualidade, destaca-se a preocupação do consumidor com a qualidade do atendimento e tempo de espera em fila.

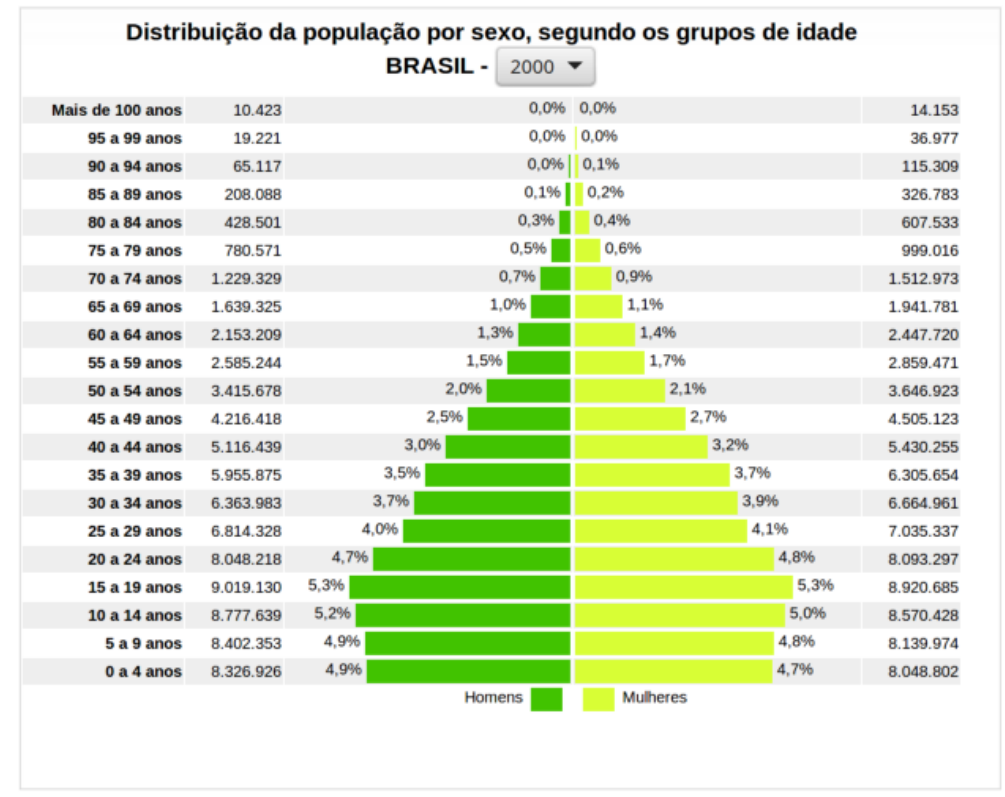

Figura 1: Pirâmide etária do Brasil em 2000. Fonte: IBGE [9].

Por outro lado, o setor farmacêutico tem sido melhor avaliado pelos consumidores do que setores tradicionais, como o de hotéis e o varejo alimentar. Contudo, as compras em farmácias não são vistas pela população como algo prazeroso. A escolha pela farmácia é realizada basicamente pelo serviço de atendimento oferecido, além do preço e facilidades de pagamento.

Dessa forma, a necessidade pela melhoria das operações das farmácias e drogarias tornam-se uma constante. O presente estudo utiliza a simulação por eventos discretos para dimensionar o número de atendentes em uma unidade de uma rede de farmácias. O método pode ser replicado às outras unidades. O objetivo consiste em relacionar do número de atendentes com o nível de serviço de atendimento, buscando possíveis economias de recursos e mantendo o padrão de excelência em atendimento.

Dessa maneira, construiu-se um modelo de simulação capaz de avaliar o 


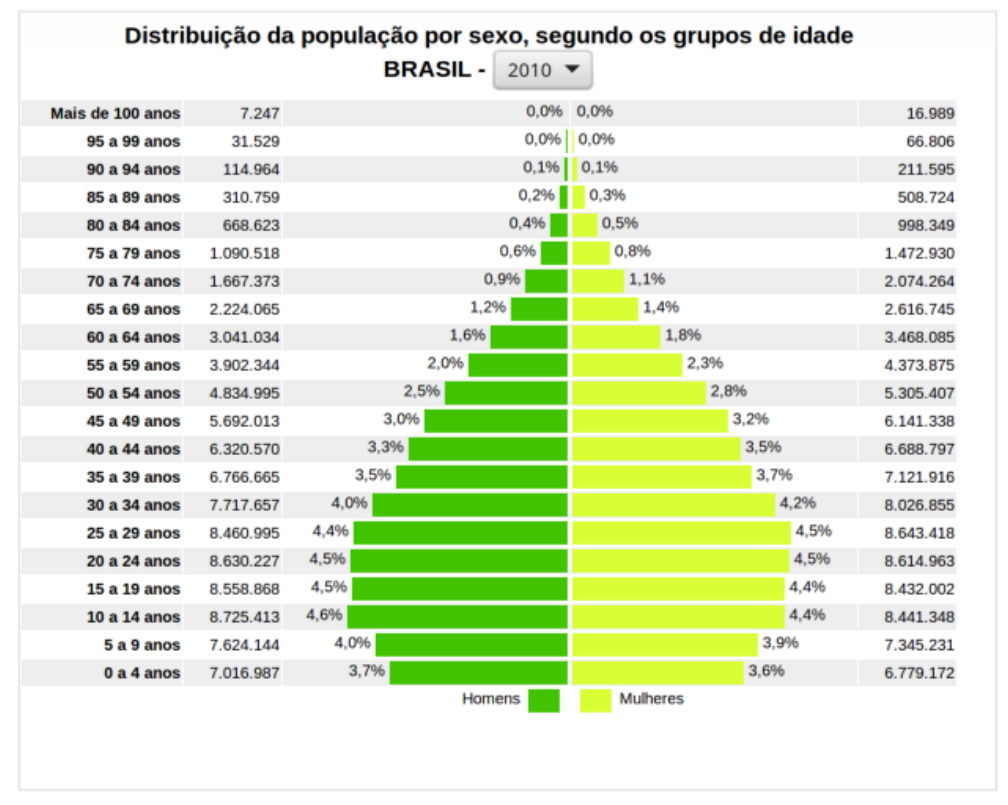

Figura 2: Pirâmide etária do Brasil em 2010. Fonte: IBGE [9].

comportamento do sistema em função do número de atendentes, tempo de atendimento, tempo de fila, tendo como premissa o foco da rede de drogaria na qualidade de atendimento ao seu cliente. Neste aspecto, o trabalho engloba outros estudos que avaliam as características de qualidade em serviços de saúde por meio de simulação, mas com um foco diferenciado ao se tratar da análise em uma rede de drogarias.

Este artigo está organizado da seguinte forma: na seção 2 examina-se a literatura recente sobre simulação por eventos discretos e aspectos sobre a qualidade em serviços no setor de saúde. Na seção3 descreve-se o problema a ser analisado. Na sequência, na seção 4 a metodologia do trabalho é detalhada, seguida na seção 5 por uma análise dos resultados obtidos por meio da simulação. Em 6 são apresentadas as conclusões, limitações do estudo e trabalhos futuros.

\section{REVISÃO DA LITERATURA}

Atualmente, a simulação é uma das técnicas mais utilizadas na Pesquisa Operacional e na Administração [10]. Modelos de simulação são utilizados para analisar um novo sistema antes da sua implantação ou compreender e melhorar a operação de um sistema já existente. Esses modelos buscam repetir no mundo simbólico o mesmo comportamento que o sistema apresentaria no mundo real com as mesmas condições, tornando possível a tomada de decisões [11] [6]. A simulação por eventos discretos é orientada por eventos, ou seja, variáveis de estado evolucionam por eventos discretos no tempo. Os estudos compõe três fases: concepção e formulação do modelo, implementação do modelo e análise dos resultados do modelo [12] [13].

Nesse sentido, a simulação pode ser um aliado no desenvolvimento e detalhamento de componentes de um serviço que sejam cabíveis às suas especificidades e 
exigências. A importância do tema recai no fato de que este setor vem em crescente expansão, tanto em representatividade econômica quanto geração de empregos. Atualmente há grande demanda por variados serviços e de melhor qualidade. De acordo com a NBR ISO 9000:2005, serviços são atividades desempenhadas pela interface entre o fornecedor e o cliente. A avaliação que o cliente faz de um serviço se dá por meio da comparação entre o que o cliente esperava do serviço e o que ele recebeu [5]. Este conceito de qualidade é subjetivo e envolve várias dimensões, dentre as quais pode se destacar a consistência, ou seja, a manutenção de um padrão de atendimento, o tempo de atendimento e o custo.

$\mathrm{Na}$ indústria farmacêutica, pesquisas apontam o pouco uso de modelagem matemática para análises e a pouca experiência dos profissionais, o que pode comprometer tentativas de melhoria da eficiência. Aplicações de tecnologia consistem na aquisição de sistemas de planejamento de necessidades materiais (Material Requirement Planning - MRPs) e investimento em softwares de previsão de demanda. Destacam-se as potencialidades de formulações por modelos de Pesquisa Operacional para maiores ganhos de produtividade e eficiência nos processos [14].

A modelagem por eventos discretos contribui para a compreensão de sistemas, permitindo potenciais aplicações de análises acerca das entidades e processos envolvidos [15]. A pesquisa realizada em um hospital avalia formas de reduzir do tempo de espera para recebimento de medicação e apontam mudanças, como alteração do local do estoque principal para locais adequados ao atendimento e a alteração do número de funcionários reduzindo significativamente o tempo de espera de pacientes por medicamentos. Estudos na indústria farmacêutica [16] destacam o uso combinado de simulação por eventos discretos integrado a métodos como PERT, CPM para avaliar a complexidade das relações sistêmicas entre os mais diversos agentes e suas implicações. Evidências no setor farmacêutico apontam a melhora do uso de recursos após a implementação do sistema de simulação para avaliar problemas de estoque dependentes de contratos estabelecidos entre fabricante e fornecedor [17].

A literatura também apresenta soluções de nível estratégico baseadas em simulação e otimização para o redesenho da rede de atacadistas do setor farmacêutico [3]. A simulação de eventos discretos é usada para avaliar os custos operacionais e o nível de atendimento ao cliente e o impacto do redesenho nas atividades diárias do atacadista. A análise dos atrasos é um dos indicadores mais importantes para a empresa, pois pode impactar o nível de serviço. Portanto, os resultados da simulação ajudam a entender os ajustes que podem ser feitos para a solução ser implementada.

Dentre os principais desafios na simulação destacam-se questões relacionadas ao comportamento humano. A complexidade do comportamento humano pode trazer novos aspectos e elementos que distorcem a simulação [18], por mais efetivo que seja a coleta de dados e a modelagem do problema. Alguns pontos são mais críticos, tal como a pessoa estar "a vontade" no local e expressar determinadas atitudes que expressem suas decisões de forma a estarem relacionadas a questões como o tamanho da fila ou o baixo nível de serviço. A seção 3 descreve o contexto abordado. Um dos fatores que não foi possível considerarmos no trabalho de forma eficiente é a forma como os operadores se organizam no trabalho e suas percepções 
pessoais. Embora o desconhecimento desta variável possa influenciar o resultado do modelo, utilizamos um nível de abstração que sugere uma abordagem generalista, com informações para análises posteriores.

\section{O CONTEXTO E O PROBLEMA}

A farmácia utilizada como base no estudo faz parte de uma rede consolidada e presente na cidade de Belo Horizonte, com mais de 180 lojas. A empresa evidencia como foco o atendimento ao cliente e a garantia de variedade de estoque, assim, comercializa não só medicamentos, mas uma grande gama de produtos de conveniência. A rede dedica um alto número de atendentes em todas as suas unidades, assim como a presença em vários pontos da cidade para atender prontamente os clientes e evitar o tempo de fila, tendo em vista a sua política corporativa de máxima satisfação do cliente no serviço de atendimento.

Na unidade analisada, 11 vendedores e 9 caixas se dividem em 3 turnos para atender a média de 500 compras por dia. Os vendedores atendem tanto no balcão, para a venda de medicamentos com receita, quanto no salão (prateleiras), para os produtos de conveniência, cosméticos ou medicamentos de venda livre. Assim, a compra do consumidor pode ocorrer de duas formas. A Figura 3 representa o Diagrama Ciclo de Atividades para o caso da farmácia.

1: O cliente chega e vai até o balcão para ser atendido, fica em fila até um vendedor o atender, espera o vendedor pegar seus remédios, vai até a fila do caixa para pagar, espera em fila até caixa liberar, faz pagamento e vai embora.

2: O cliente chega e seleciona produtos de venda livre no salão (prateleiras), vai até a fila do caixa para pagar, espera em fila até caixa liberar, faz pagamento e vai embora.

O objetivo da simulação é dimensionar o número de atendentes na farmácia, analisando a sua relação com a agilidade no tempo de atendimento. Dessa maneira, construiu-se um modelo capaz de avaliar o comportamento do sistema em função do número de atendentes, tempo de atendimento, tempo de fila, tendo como premissa o foco da rede de drogaria na qualidade de atendimento ao cliente. Os métodos de coleta de dados e outras especificidades do modelo são apresentadas a seguir, na Metodologia.

\section{METODOLOGIA}

As características do objeto de estudo, em que nota-se um ambiente com alta estocasticidade e um conjunto de normas flexíveis à situação corrente no estabelecimento, evidenciam as vantagens da abordagem via simulação de eventos discretos. Portanto, torna-se, então, prático estudar a dinâmica do local de forma objetiva. Há de se considerar o contexto organizacional e estratégia da empresa para direcionar a metodologia de estudo, desta forma, estabelece-se a decisão de 


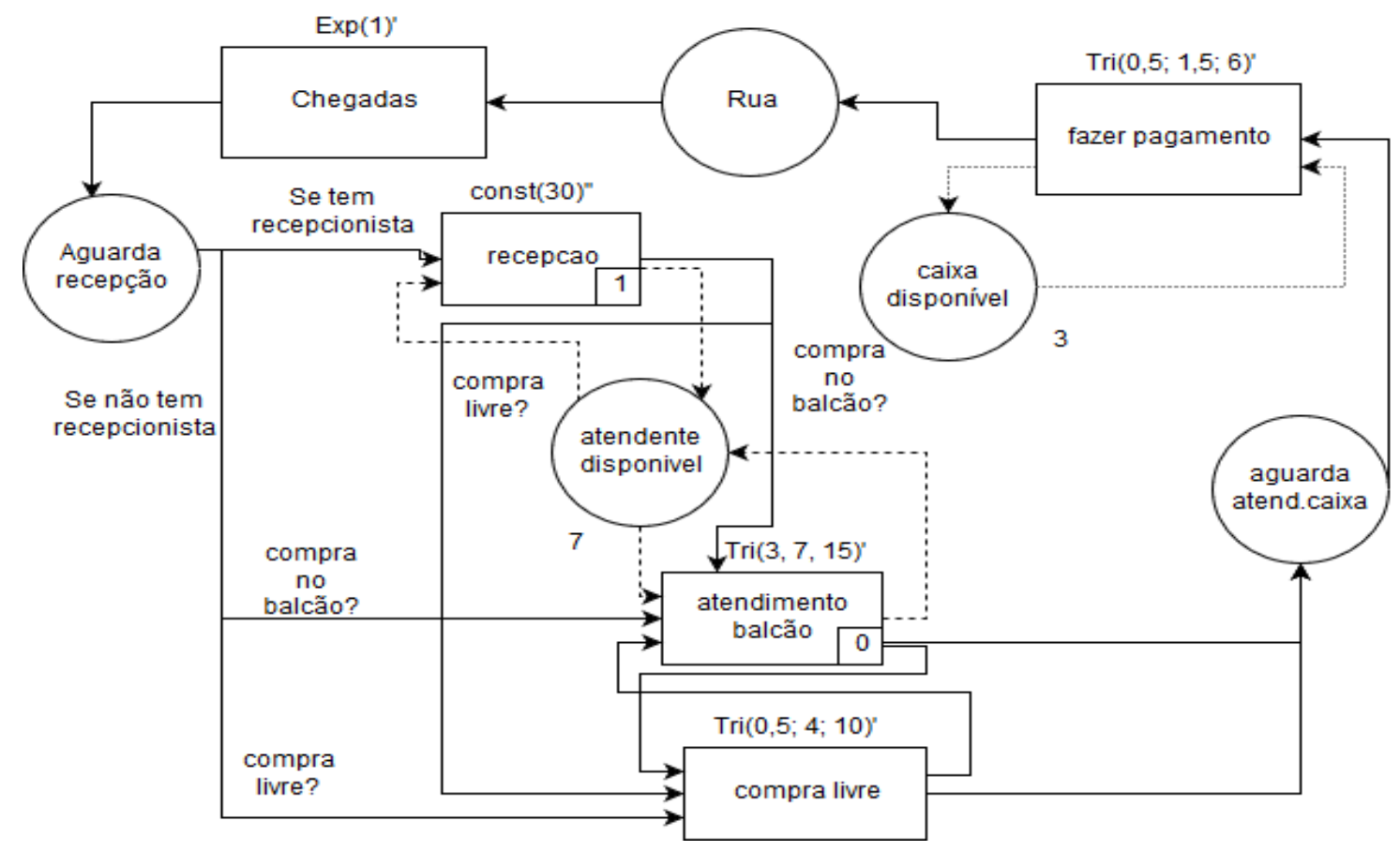

Figura 3: Diagrama de Ciclo de Atividades para o atendimento na farmácia

abordar o tema através da análise e observação, focado nos critérios de aplicação da estratégia de atendimento da rede nas diversas conjunturas possíveis durante o horário de funcionamento de uma drogaria. Posto isto, a construção das informações se deu através de coleta de dados e observação para a construção do modelo que permite a simulação do estabelecimento analisado. O modelo elaborado se encontra descrito por pseudo-algoritmo, e a subsequente simulação de cenários são apresentados a seguir.

Os dados foram obtidos por meio de visitas ao local de estudo, realizadas durante os intervalos de funcionamento com maior probabilidade de tempos de filas consideravelmente longos, relacionados aos momentos de maior recepção de clientes (horários de pico). Dada a similaridade da operação rotineira, a equipe optou por fazer a coleta do dados de funcionamento do sistema por um período de três dias de observações, em um ponto de atendimento da rede de farmácias, no entanto, um número de amostras maior pode conferir mais representatividade dos dados. Duas gerentes de duas unidades distintas responderam a uma entrevista, feita para compreender os procedimentos de atendimento da empresa e atestar a conciliação entre suas unidades e a aplicação das condutas de acolhimento de clientes. Os registros foram analisados estatisticamente de forma a fornecer medidas de posição e dispersão, resultando nas distribuições utilizadas no estudo. A Tabela 1 exprime as distribuições e seus respectivos parâmetros obtidos para as atividades modeladas na simulação proposta. Distribuições triangulares foram obtidas de entrevistas com funcionários experientes.

Os cenários foram construídos de forma a estudar possíveis situações extremas. Em um estudo de mesma autoria, foi observado que o recurso crítico no 
Tabela 1: Modelo dos dados coletados em uma unidade da rede de farmácias

\begin{tabular}{llll} 
Eventos & Distribuição de probabilidades & Parâmetros & Unidade \\
\hline Chegadas de clientes na farmácia (horário de pico) & exponencial & 1 & (cliente/min) \\
Chegadas de clientes na farmácia (normal) & exponencial & 0.33 & $(\mathrm{cliente} / \mathrm{min})$ \\
Tempo de atendimento no balcão & triangular & {$[3,15,7]$} & $(\mathrm{min})$ \\
Tempo de compra no salão & triangular & {$[0.5,4,10]$} & $(\mathrm{min})$ \\
Tempo de atendimento no caixa & triangular & {$[0.5,6,1.5]$} & $(\mathrm{min})$
\end{tabular}

estabelecimento são os operadores de caixa. Portanto, os cenários apresentam valores aumentados de taxa de chegadas em relação ao que foi observado e apresentado na Tabela 1. Sendo considerados os valores de taxas de chegada $(\alpha)$ em horário de pico: $\alpha=2.0$ e, $\alpha=0.2$ em um momento de baixa demanda. Há duas possíveis variações de parâmetros para a simulação, que dizem respeito ao horário de funcionamento (horário de pico ou não) e número de atendentes de caixa na farmácia. As combinações entre essas variações foram avaliadas, dando origem a quatro cenários para os experimentos de simulação. No primeiro cenário, considerou-se que há todos os três operadores de caixa presentes na loja, bem como uma baixa taxa de chegada, simulando uma situação com baixa probabilidade de formação de filas. No segundo cenário, variou-se a taxa de chegada, simulando uma hora de pico com o número de caixas funcionando igual a 3. O terceiro cenário considera baixo movimento, entretanto com apenas 2 caixas funcionando. Por fim, o quarto cenário avalia a situação em que um atendente está ausente dos caixas durante uma hora com taxa de chegadas alta.

A simulação foi implementada utilizando o Simpy [19] [20], uma estrutura para ambientes de simulação de eventos discretos, utilizando a linguagem de programação Python.

\section{ANÁLISES E RESULTADOS}

O modelo de negócio da empresa baseia-se na comodidade que o cliente tenha durante o processo de compra, o que é representado pela rapidez nos atendimentos em cada um dos processos da farmácia. Assim, os cenários buscam avaliar as repercussões de alterações na estrutura de atendimento da farmácia sobre o desempenho esperado por esta política da empresa.

O teste do modelo verificou que nos cenários semelhantes ao que fora observado nas visitas técnicas, neles, os tempos em filas de cada processo de atendimento são baixos. Concluindo, assim, que o modelo produz resultados com boa aproximação aos dados coletados na unidade estudada, uma vez que parte da política de atendimento da empresa é de garantir um baixo tempo em fila por cliente. Estes cenários assim como os que apresentam valores teste se encontram detalhados a seguir.

Cenário 1: Hora de baixo movimento, com todos os atendentes presentes. Foram necessárias 31 replicações de simulação para obter a precisão desejável ao nível de confiança estatística de $95 \%$. Os resultados de tempo e número de clientes em fila e no sistema são apresentados nas Figuras 4 e 5 e Tabela 2. Neste 
cenário, os tempos em fila são próximos de zero, havendo somente alguns casos em que o simulador cria uma rodada com um tempo em fila do caixa significativo, os tempos em fila no farmacêutico foram iguais a zero em todas as rodadas. Como observado, a diretriz de atendimento reduz drasticamente as filas na farmácia, e a taxa de utilização dos recursos é alta, em torno de $92 \%$.

Tabela 2: Indicadores de desempenho do Cenário 1 da simulação

\begin{tabular}{lrl}
\hline Indicador de desempenho & Valor & Unidade \\
\hline Tempo médio em fila para o farmacêutico & $\approx 0$ & min \\
Tempo médio em fila para o caixa & 0,02 & min \\
Média de clientes na farmácia & 2,76 & cliente \\
Média do tempo no sistema & 10,47 & min \\
Número médio de clientes na fila do farmacêutico & 0 & cliente \\
Número médio de clientes na fila do caixa & 0,02 & cliente \\
Utilização média do sistema (em atendimento) & 92,61 & percentual(\%) \\
Tempo médio global das filas & 0,02 & min \\
\hline
\end{tabular}

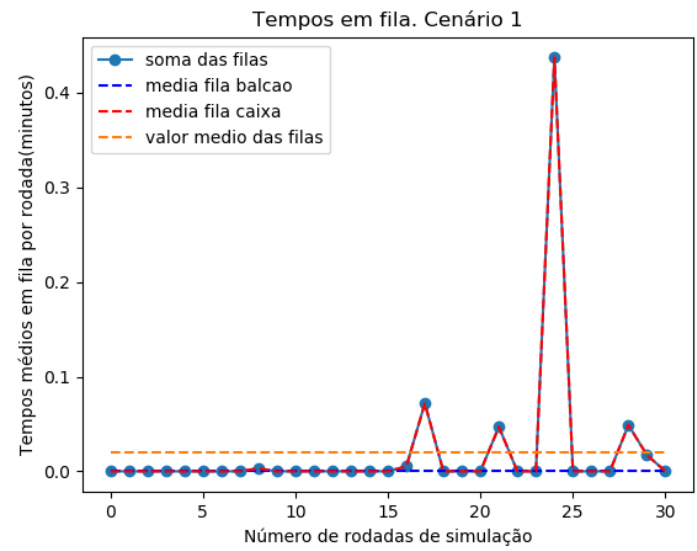

Figura 4: Cenário 1: tempo em fila

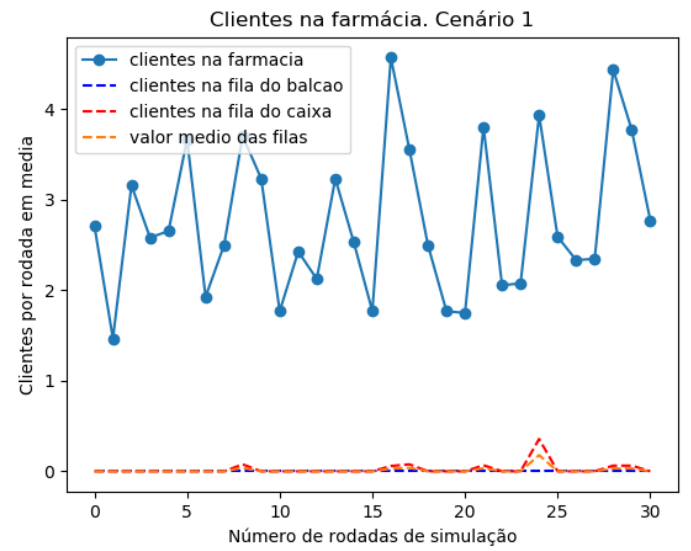

Figura 5: Cenário 1: clientes em fila

Cenário 2: Hora de pico, com todos os operadores de caixa na loja. 129 replicações de simulação foram realizadas para conseguir obter a precisão desejável ao nível de confiança estatística de 95\%. Os resultados de tempo e número de clientes em fila e no sistema são apresentados nas Figuras 6 e 7 e Tabela 3 . Nota-se que o ritmo de chegada tem impacto significativo nos tempos de atendimento e no número de pessoas em fila. O estudo de um cenário considerado extremo(o dobro de chegadas por minuto na hora de pico), leva a uma taxa de utilização moderada de $56,19 \%$, pode-se supor que a capacidade ainda atende a política de atendimento ao cliente, uma vez que esta taxa de chegadas é considerada inesperada e bem acima do observado pelos autores.

Cenário 3: Hora com pouco movimento, sem um dos atendentes no caixa. Foram necessárias 32 replicações de simulação para obter a precisão desejável ao nível 
Tabela 3: Indicadores de desempenho do Cenário 2 da simulação

\begin{tabular}{lrl}
\hline Indicador de desempenho & Valor & Unidade \\
\hline Tempo médio em fila para o farmacêutico & 11,61 & min \\
Tempo médio em fila para o caixa & 2,73 & min \\
Média de clientes na farmácia & 37,79 & cliente \\
Média do tempo no sistema & 17,70 & min \\
Número médio de clientes na fila do farmacêutico & 6,02 & cliente \\
Número médio de clientes na fila do caixa & 4,17 & clientes \\
Utilização média do sistema (em atendimento) & 56,19 & percentual(\%) \\
Tempo médio global das filas & 7,170 & min \\
\hline
\end{tabular}

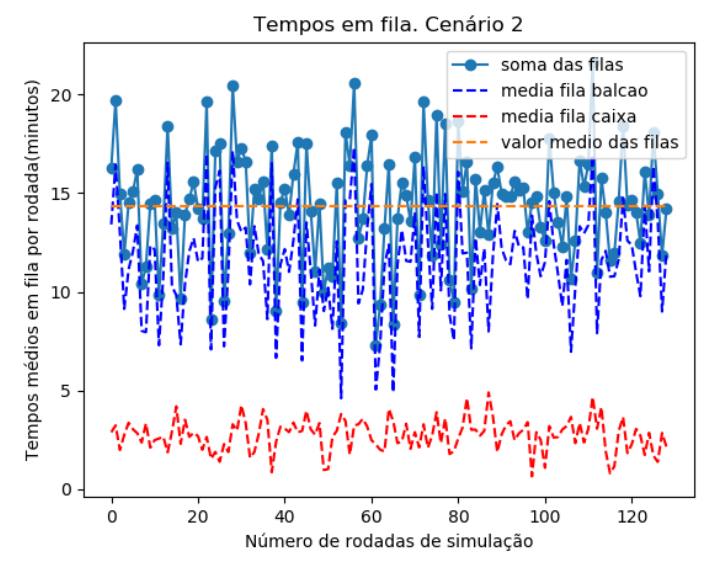

Figura 6: Cenário 2: tempo em fila

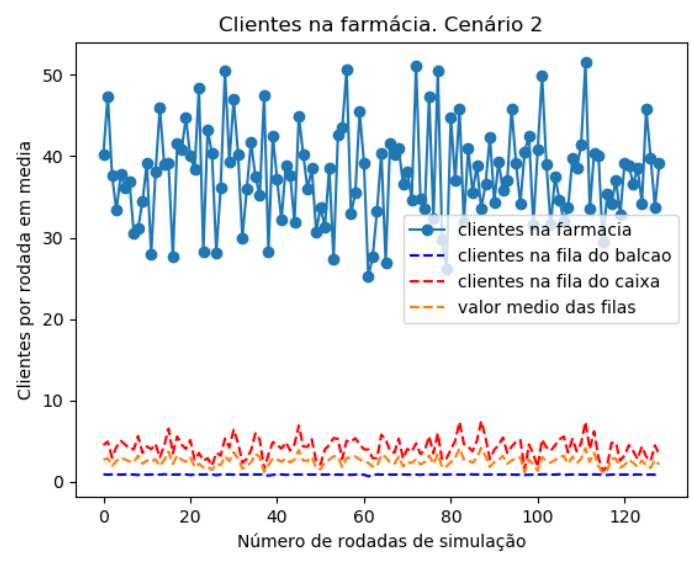

Figura 7: Cenário 2: clientes em fila

de confiança estatística de $95 \%$. Os resultados de tempo e número de clientes em fila e no sistema são apresentados nas Figuras 8 e 9 e Tabela 4 . Neste caso, os tempos em fila são baixos, dessa forma, o algoritmo calcula os tempos de fila individuais como próximos de 0 , especialmente no atendimento no balcão de farmacêuticos, assim como no cenário 1. A taxa de utilização de $90 \%$ é muito alta, considerando a falta de um atendente. Com esse dado, podemos supor que o sistema foi modelado pela empresa para suportar a falta de um operador de caixa em situações de baixa demanda sem que isso altere de forma significativa o nível de serviço.

Cenário 4: Hora de pico, com um atendente a menos nos caixas da farmácia. O algoritmo executou 134 replicações de simulação para obter a precisão desejável ao nível de confiança estatística de $95 \%$. Os resultados de tempo e número de clientes em fila e no sistema são apresentados nas Figuras 10 e 11 e Tabela 5. No caso em que a demanda é alta, neste caso, o dobro do esperado para uma hora de pico e, ainda, falta um atendente no caixa, podemos notar que o tempo de fila nos caixas aumenta para um nível bastante alto se comparado com os cenários com demanda baixa, contudo, dentro de uma faixa de tempo que a empresa considera dentro dos limites de satisfação esperados, abaixo de 5 minutos. Porém, a fila no balcão aumenta consideravelmente, de aproxima- 
Tabela 4: Indicadores de desempenho do Cenário 3 da simulação

\begin{tabular}{lrl}
\hline Indicador de desempenho & Valor & Unidade \\
\hline Tempo médio em fila para o farmacêutico & 0,00083 & $\mathrm{~min}$ \\
Tempo médio em fila para o caixa & 0,11 & $\mathrm{~min}$ \\
Média de clientes na farmácia & 2,88 & cliente \\
Média do tempo no sistema & 10,55 & min \\
Número médio de clientes na fila do farmacêutico & 0,02 & cliente \\
Número médio de clientes na fila do caixa & 0,104 & cliente \\
Utilização média do sistema (em atendimento) & 90,28 & percentual(\%) \\
Tempo médio global das filas & 0,054 & min \\
\hline
\end{tabular}

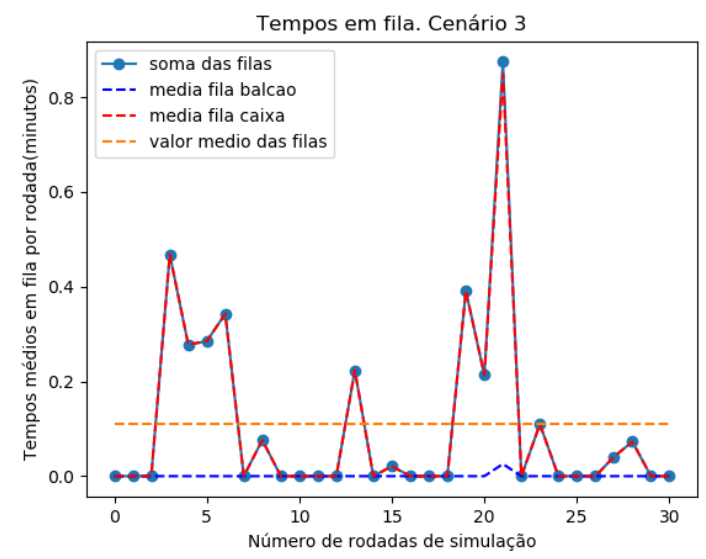

Figura 8: Cenário 3: tempo em fila

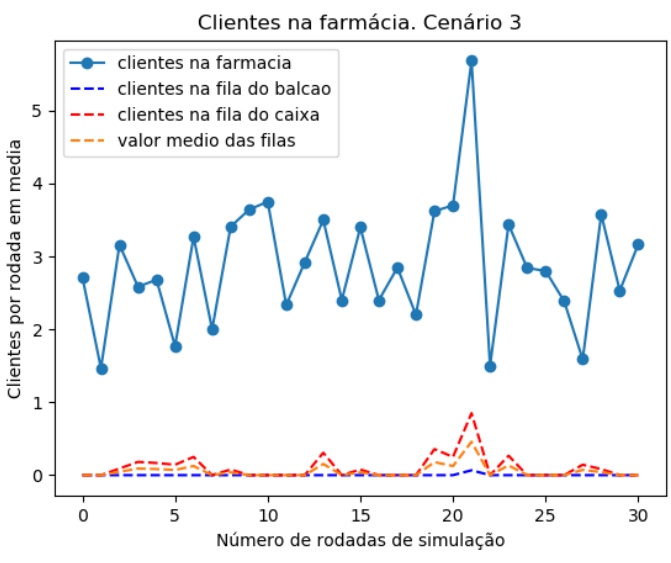

Figura 9: Cenário 3: clientes em fila

damente 0 minutos nas situações para 11,90 minutos. Dessa forma, o sistema tem uma redução considerável na taxa de utilização dos recursos observados. O algoritmo calculou um percentual de utilização de 48,80\%, assim, pode-se considerar uma utilização intermediária da capacidade de atendimento. Neste cenário podemos notar que o sistema de atendimento mantém um padrão de serviço que retém uma razoável utilização dos recursos mesmo em uma situação de horário de pico mais elevado que o valor observado e com 1 atendente a menos no caixa da loja.

Tabela 5: Indicadores de desempenho do Cenário 4 da simulação

\begin{tabular}{lrl}
\hline Indicador de desempenho & Valor & Unidade \\
\hline Tempo médio em fila para o farmacêutico & 11,90 & min \\
Tempo médio em fila para o caixa & 4,82 & min \\
Média de clientes na farmácia & 39,16 & clientes \\
Média do tempo no sistema & 18,72 & min \\
Número médio de clientes na fila do farmacêutico & 5,99 & clientes \\
Número médio de clientes na fila do caixa & 6,82 & clientes \\
Utilização média do sistema (em atendimento) & 48,80 & percentual(\%) \\
Tempo médio global das filas & 8,357 & min \\
\hline
\end{tabular}




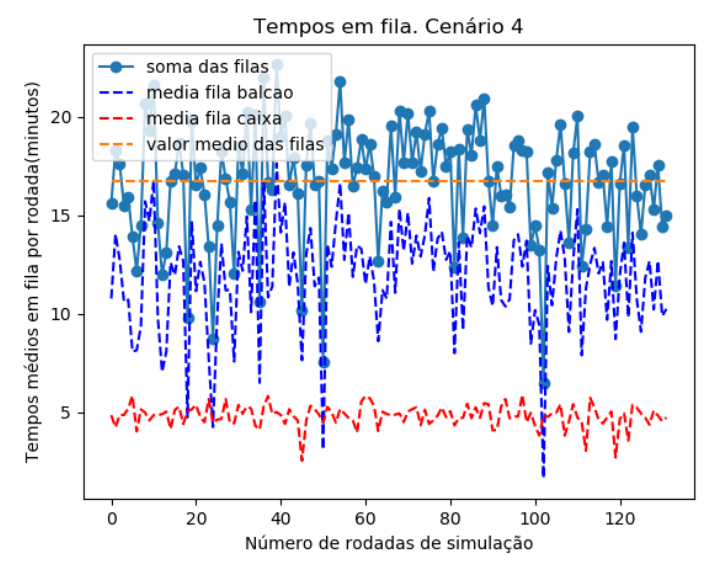

Figura 10: Cenário 4: tempo em fila

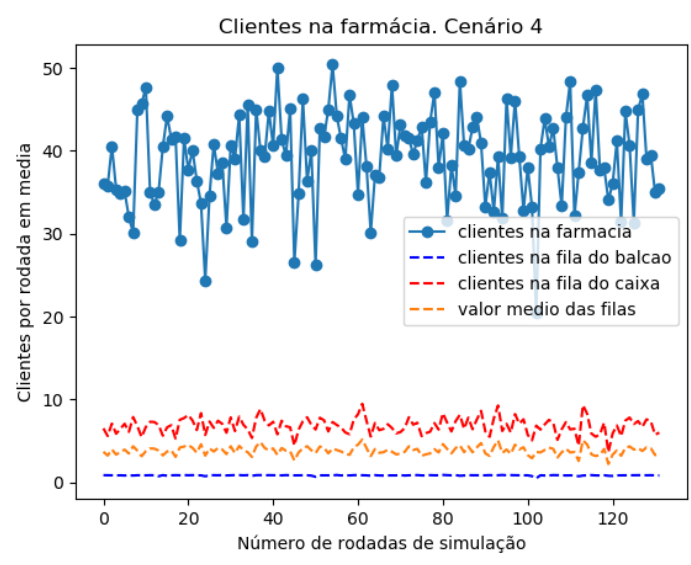

Figura 11: Cenário 4: clientes em fila

Para o estudo, foram considerados como parâmetros a serem variados o número de operadores em caixa e a taxa de chegada de clientes em horário de pico, considerando um valor bem acima do observado em campo. O cenário 1 representa uma situação bem semelhante ao observado, servindo como referência para o comportamento padrão da loja nos intervalos com pouca demanda. O cenário 3 apresenta a variação no número de caixas em funcionamento e os resultados observados nesse cenário mostram que a política de atendimento da empresa, como informado pela gerência, comporta a redução de um operador e mantendo o nível de serviço desejado. Os cenários 2 e 4 apresentam a variação na taxa de chegada de clientes por minuto. Nestes cenários são observados os maiores desvios em relação ao observado durante as visitas, atestando assim, de fato que um cenário considerado extremo gera impactos significativos no sistema de atendimento, e na taxa de utilização da capacidade, observando a formação de filas longas no balcão de fármacos controlados. Ademais, nota-se que o impacto da taxa de chegadas é aumentado pelo fato de haver um funcionário a menos no caixa.

Uma análise dos quatro cenários mostra que o atendimento ao cliente foi concebido para reduzir ao máximo os tempos em fila e, que de fato o sistema se comporta da maneira esperada pelos seus gestores quando o sistema está num contexto dentro da capacidade de recepção média observada, uma vez que em todas as situações com poucas chegadas por minuto os clientes passaram pouco tempo em fila na média. Em outros estudos dos mesmos autores, a análise de cenários mais conservadores atesta uma taxa de ocupação acumulada dos recursos acima de $80 \%$ em todos os cenários. Uma indicação da qualidade é o nível de serviço estar acima de $90 \%$ nos cenários de baixo movimento e próximos a $50 \%$ nos casos com uma taxa de chegadas muito elevada. Assim, nos variados cenários testados, um cliente padrão admite esperar durante alguns minutos e ainda considera que recebeu um bom atendimento. Sendo improvável uma situação com tempos elevados aguardando atendimento.

\section{CONSIDERAÇÕES FINAIS E TRABALHOS FUTUROS}




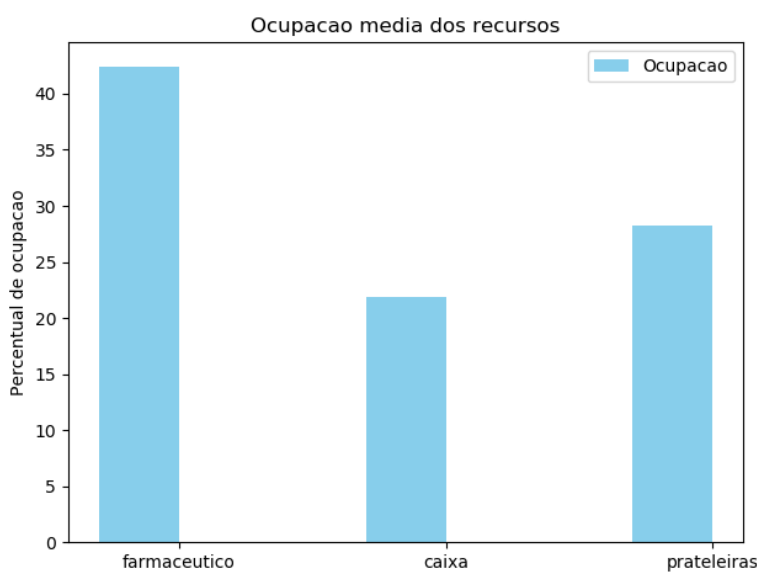

Figura 12: Cenário 1

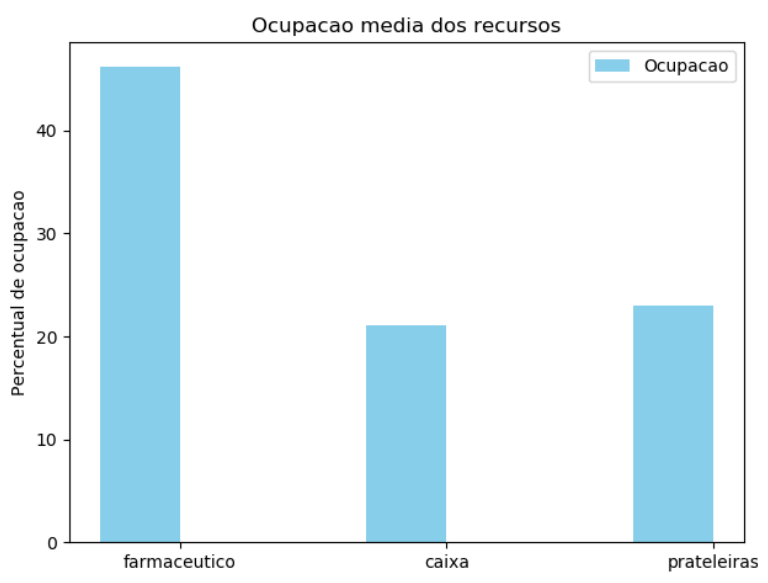

Figura 14: Cenário 3

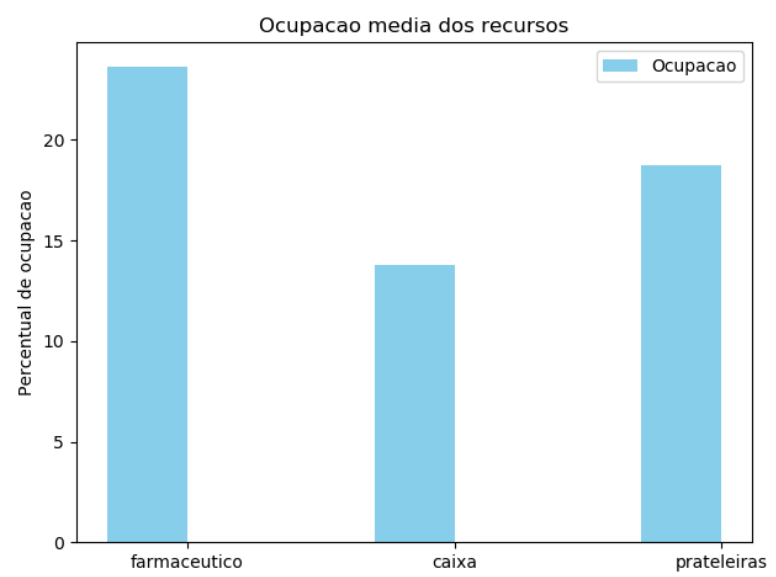

Figura 13: Cenário 2

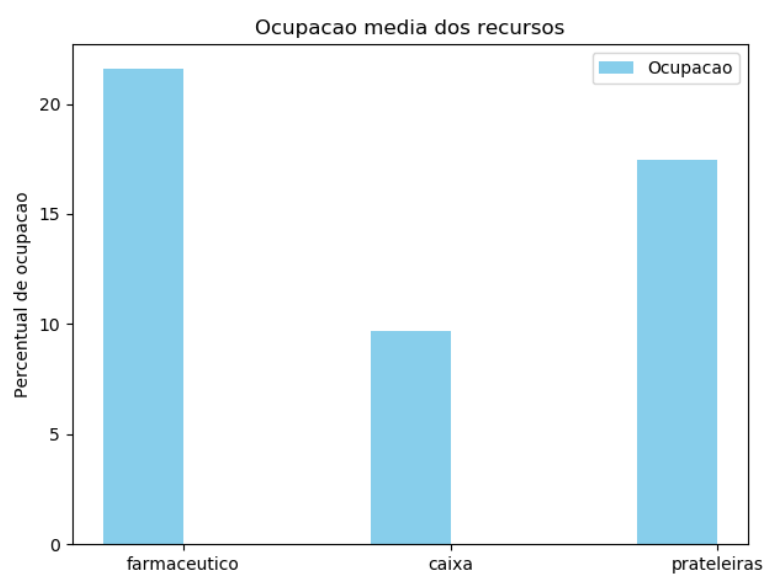

Figura 15: Cenário 4

Figura 16: Figuras 10 - 13: percentuais de utilização por recurso comparado ao tempo total na farmácia.

O presente estudo teve o objetivo de caracterizar o dimensionamento do número de atendentes em uma unidade de uma rede de drogarias, apoiado na Simulação por Eventos Discretos. A construção de um modelo representativo do sistema permitiu avaliar o seu comportamento em função do número de atendentes, tempo de atendimento, tempo em filas e taxa de ocupação dos recursos. Através da análise de quatro cenários para a dinâmica de funcionamento do objeto de estudo, foi constatado a ocorrência de pouco tempo de fila e alto nível de serviço nos cenários conservadores e mais reduzidos nos cenários extremos. A discussão apresentada visa conciliar a melhoria do sistema com as políticas corporativas da rede, que têm como objetivo a satisfação do cliente durante o atendimento.

A modelagem do problema buscou ser fiel às dinâmicas que influenciam os processos de compra na rede estudada. Uma limitação imposta a análise está na ausência na simulação de aspectos relevantes ao modelo desta farmácia, como a possível atuação do gerente nos processos de atendimento ao cliente, juntamente 
com os atendentes. Essa participação foi desconsiderada por abranger a organização do trabalho da drogaria, o que exigiria uma observação muito aprofundada do dia a dia do gerente para modelar corretamente a sua atuação e entender em quais situações ele participa do atendimento na função do operador. Tais observações fogem do escopo de análise aqui proposto. Apesar destes pontos, o estudo complementa a literatura que avalia o aspecto de qualidade em serviços de saúde por meio de Simulação, com um foco diferenciado ao se tratar da análise em uma rede de drogarias.

Dentre as contribuições, o estudo promove à rede de drogarias um ambiente de discussão que pode representar mudanças culturais e financeiras, uma vez que ilustra cenários que podem instigar a alteração de políticas da empresa de números de funcionários e estratégia de atendimento. Tal potencial evidencia oportunidades para se expandir o estudo para o contexto de outras unidades da rede. Assim, a análise quanto ao dimensionamento de funcionários para as 180 lojas amplia perspectivas de diferentes arranjos possíveis para a dinâmica de atendimento da empresa, que pode gerar resultados significativos em termos de custos e melhoria do nível de serviço. Futuros estudos podem ainda ampliar as bases de pesquisa, passando a tomar parte do modelo outros meios de consumo que têm se tornado cada vez mais difundidos na rede estudada, como as vendas online, em que a jornada do cliente e sua percepção de qualidade se torna mais diversificado.

\section{AGRADECIMENTOS}

Os autores agradecem aos revisores pelas análises e sugestões de melhoria do texto contribuindo para o aumento da qualidade deste artigo. 


\section{REFERÊNCIAS BIBLIOGRÁFICAS}

[1] DALFOVO MICHAEL SAMIR; LANA, R. A. S. A. Métodos quantitativos e qualitativos: um resgate teórico. revista interdisciplinar científica aplicada. Revista interdisciplinar científica aplicada, Unibes, v. 12, p. 1-13, 2008. 1]

[2] ALmEIDA, J. F. F. Simulação por Eventos Discretos. Teoria e Prática. [S.l.], 2016. 1

[3] MARTINS, S. et al. An optimization-simulation approach to the network redesign problem of pharmaceutical wholesalers. Computers 83 Industrial Engineering, Elsevier, v. 106, p. 315-328, 2017. 1, 4

[4] DADOS. Conselho Federal de Farmácia. 2016. http://www.cff.org.br/ pagina.php?id=801\&titulo=Boletins/. Acessado: 2018-11-21. [1]

[5] TOLEDO, J. C. d. e. a. Qualidade: gestão e métodos. Rio de Janeiro: LTC, 2013. 2, 4

[6] CHWIF, L.; MEDINA, A. C. Modelagem e simulação de eventos discretos: teoria Er aplicações. [S.1.]: Autores, 2006. 2, 3

[7] BARAT, I.; ANDREASEN, F.; DAMSGAARD, E. M. S. The consumption of drugs by 75 -year-old individuals living in their own homes. European Journal of Clinical Pharmacology, v. 56, n. 6, p. 501-509, Sep 2000. ISSN 1432-1041. Disponível em: <https://doi.org/10.1007/s002280000157>. 2

[8] ZANON RODRIGO RAFAEL; MORETTO, A. C. R. R. L. Envelhecimento populacional e mudanças no padrão de consumo e na estrutura produtiva brasileira. Revista Brasileira de Estudos de População, rebep, v. 30, n. 45-67, 2013. 2

[9] IBGE. Pirâmide etária - Sinopse dos resultados do Censo. 2019. https:// censo2010.ibge.gov.br/sinopse/webservice. Acessado: 2019-05-01. 2 , 3

[10] BORGES, P. C. R. Métodos quantitativos de apoio à bibliometria: a pesquisa operacional pode ser uma alternativa? Ciência da informação, SciELO Brasil, v. 31, n. 3, 2002. 3

[11] CHWIF, L. Redução de modelos de simulação de eventos discretos na sua concepção: uma abordagem causal. São Paulo, 1999. 3

[12] BALMER, D. W.; PAUL, R. J. Casm-the right environment for simulation. Journal of the Operational Research Society, Springer, v. 37, n. 5, p. 443-452, 1986. 3

[13] PAUL, R. J.; BALMER, D. W. Simulation Modelling. [S.l.]: Chartwell-Bratt, London, 1993. 3 
[14] MESQUITA, M. A. d.; SANTORO, M. C. Analysis of models and practices in production and inventory management: A case study in brazilian pharmaceutical industry. Production, SciELO Brasil, v. 14, n. 1, p. 64-77, 2004. 4

[15] COLLAZO, R. A. et al. Simulação orientada ao cliente: distribuição de medicamentos do hospital naval marílio dias. XLI SBPO-Simpósio Brasileiro de Pesquisa Operacional, v. 41, 2009. 4

[16] SILVA, E. M. L. Desenvolvimento de um modelo de simulação para apoio ao gerenciamento de projetos de desenvolvimento de medicamento genérico em uma empresa farmacêutica. Uninove: Dissertação, 2015. 4

[17] ZHAO, H. e. a. Fee-for-service contracts in pharmaceutical distribution supply chains: design, analysis, and management. Manufacturing $\& 5$ Service Operations Management, Informs, v. 14, n. 4, p. 685-699, 2012. 4

[18] GREASLEY, A.; OWEN, C. Modelling people's behaviour using discrete-event simulation: a review. International Journal of Operations $\&$ Production Management, Emerald Publishing Limited, v. 38, n. 5, p. 1228-1244, 2018. 4

[19] MATLOFF, N. Introduction to discrete-event simulation and the simpy language. Davis, CA. Dept of Computer Science. University of California at Davis. Retrieved on August, v. 2, n. 2009, p. 1-33, 2008. 7

[20] SIMPY, T. Simpy: Discrete event simulation for python. Python package version, v. 3, n. 9, 2017. 7 